\title{
Modified Mini-Maze for Atrial Fibrillation with Interruption of Inferior Vena Cava
}

\author{
Bijun Xu, MD, Fan He, MD, Shiqiang Wang, MD, Huaidong Chen, MD, Weimin Zhang, MD \\ Department of Cardiovascular Surgery, Sir Run Run Shaw Hospital, Zhejiang University
}

\section{ABSTRACT}

A 57-year-old female with paroxysmal atrial fibrillation and an interrupted inferior vena cava (IVC) was referred to our hospital for radiofrequency ablation. Transseptal puncture and left atrium ablation failed through a standard IVC approach via the femoral vein due to intrahepatic interruption of IVC. We performed a modified mini-maze procedure in this patient through the left thoracic cavity under videoassisted thoracoscopic surgery (VATS). We can successfully complete pulmonary vein (PV) isolation, left atrium box isolation, cardiac ganglia ablation, Marshall ligament ablation, and coronary sinus epicardium ablation using this technique.

\section{INTRODUCTION}

The duplication of the inferior vena cava (IVC) is a rare anatomical variation that occurs during embryological development of the IVC [Giordano 1986]. This anomaly interrupts the IVC, which renders the ablation of atrial fibrillation (AF) impossible with the standard approach via IVC to the left atrium. To address the technical difficulty, we adopted the surgical bipolar radiofrequency ablation (endoscopic maze procedure) through the left chest cavity in patients with an interrupted IVC.

\section{PATIENT AND PROCEDURES}

A 58-year-old female patient was presented with recurrent palpitation for one month. Holter electrocardiography monitoring showed paroxysmal AF. She was treated with oral amiodarone for a month, but it didn't work. So, she was admitted to our hospital for catheter ablation.

Echocardiography revealed a $40 \mathrm{~mm}$ left atrium in anteroposterior diameter and $62 \%$ left ventricular ejection fraction (LVEF). During the procedure, the cardiologist could not put the catheter into the right atrium. Angiography showed interruption of the IVC. Further computed tomographic venography (CTV) showed double IVC with intrahepatic

Received fuly 22, 2021; accepted October 6, 2021.

Correspondence: Weimin Zhang, East Qingchun Road 3th, Hangzhou, Zhejiang Province, China, Telephone +8613588118160 (e-mail: zwm660@zju.edu.cn). interruption, azygos and hemiazygos vein continuation, and intrahepatic venous shunt. The hepatic vein was shown to connect the right atrium. (Figure 1)

We decided to perform Modified mini-maze via left thoracic cavity under VATS on this patient. The procedure

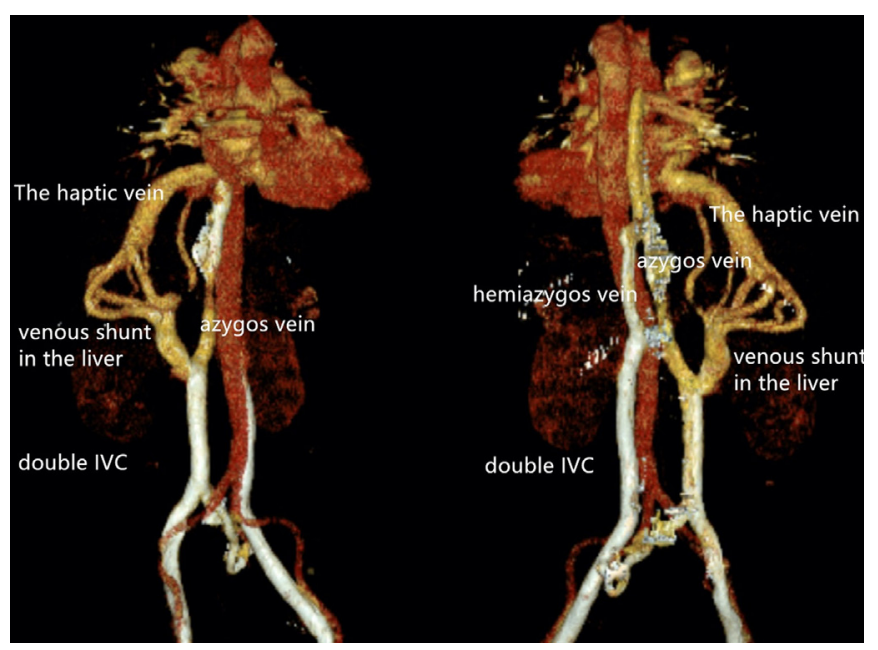

Figure 1. The CTV showed double IVC with intrahepatic interruption, azygos and hemiazygos vein continuation, and intrahepatic venous shunt. The hepatic vein was shown to connect the right atrium.

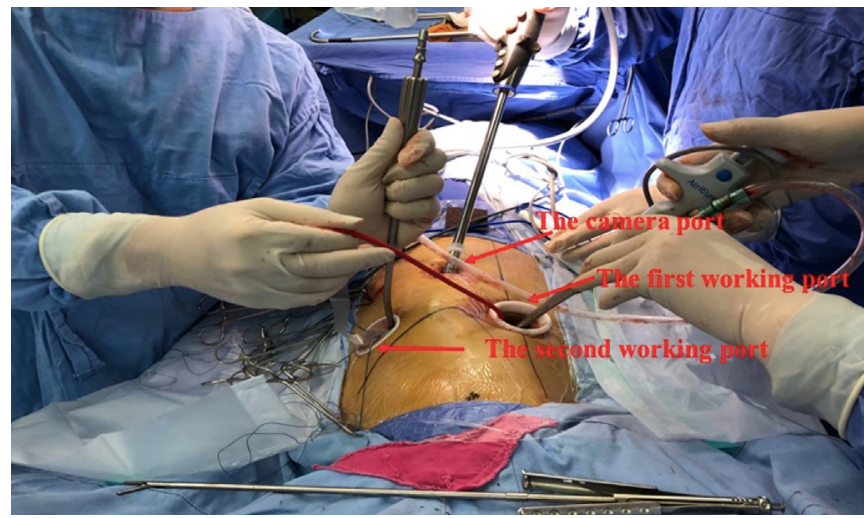

Figure 2. The procedure was performed through 3 ports in the left chest wall. The camera port $(10 \mathrm{~mm})$ was introduced in the eighth intercostal space at the subscapular angle line. The first working port ( 25 $\mathrm{mm}$ ) was introduced about $30 \mathrm{~mm}$ anterior to the subscapular angle line in the sixth intercostal space. The second working port $(10 \mathrm{~mm})$ was introduced $30 \mathrm{~mm}$ posterior to the subscapular angle line in the seventh intercostal space. 


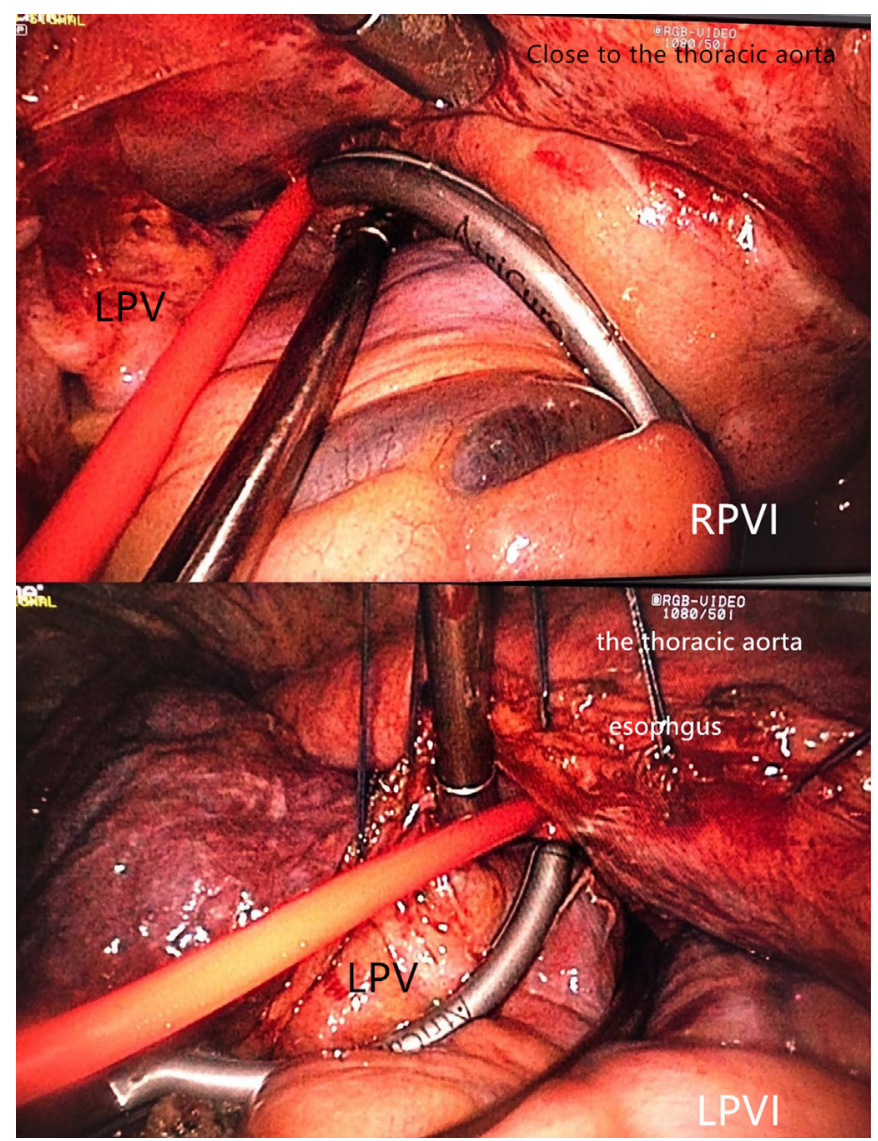

Figure 3. The right and left pulmonary vein isolation were achieved by bipolar radiofrequency ablation device.

was performed on the beating heart through 3 ports in the left chest wall. (Figure 2) The pulmonary vein isolation and ablation of the left atrium were achieved by bipolar radiofrequency ablation with AtriCure Isolator Synergy ablation clamp. (Figure 3) Ganglionic plexus ablation was completed using the AtriCure Isolator Synergy ablation pen. The left atrial appendage was excluded. The operation was very successful, and no complications occurred during or after the procedure. The patient was discharged with sinus rhythm 3 days after the procedure and taking amiodarone (100mg bid) for 6 months. Moreover, she has been well and followed up for 2 years with no recurrence of AF.

\section{DISCUSSION AND CONCLUSION}

Congenital IVC malformation reflects the complicated multi-segmental development of the IVC during embryogenesis [McClure 1925]. It is classified by Huntington and McClure with up to 14 theoretical variations [Maxwell 1928]. The most common anomalies of the IVC include the circumaortic left renal vein $(1.5 \%-8.7 \%)$, retroaortic left renal vein $(2.1 \%)$, double IVC $(0.2 \%-3 \%)$, azygos, hemi-azygos continuation of IVC $(0.6 \%)$, and isolated left-sided IVC

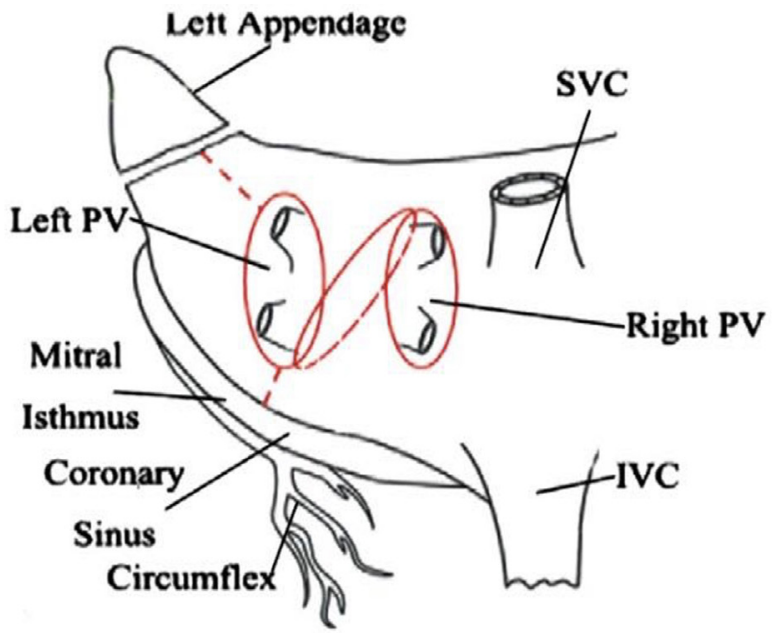

Figure 4. The linear lesion from the left inferior pulmonary vein to the right superior pulmonary vein by the bipolar radiofrequency ablation clamp. PV, pulmonary vein; SVC, superior vena cava; IVC, inferior vena cava. [Mei 2014]

(0.2\%-0.5\%) [Sahin 2017]. The double IVC, azygos and hemiazygos continuation, intrahepatic IVC interruption, and transhepatic venous shunt are rare. Here, we reported the patient with the intrahepatic shunt on the right and hemi-azygos system on the left for the venous return from extremities. The patient had the retroaortic anastomotic vein connecting 2 venous collateral pathways toward the heart. Most patients with IVC malformation are asymptomatic. The prevalence of thromboembolic disease in patients with a duplicated IVC is unknown, yet there are case reports of pulmonary embolism in those patients [Sahin 2017].

Cardiologists may use a superior vena cava (SVC) approach for transseptal puncture through the right internal jugular vein in LA ablation, but that is not a conventional choice. It is impossible to puncture the atrial septal by IVC approach in patients with IVC anomaly. The Cox Maze III procedure has been considered the "gold standard" to eliminate AF, but it is not well accepted by patients because of its invasiveness. Wolf and colleagues introduced using the thoracoscopic technique to perform Cox Maze procedure [Wolf 2005]. However, the procedures are technically challenging. It was further modified by Mei, who reported a complete thoracoscopic ablation of the LA via the left chest to treat lone atrial fibrillation [Mei 2014]. The ablation circuit was shown in Figure 4. We used Mei's method on the beating heart through 3 ports in the left chest wall. Pulmonary vein isolation and ablation of the left atrium were achieved by bipolar radiofrequency ablation. Ganglionic plexus ablation was completed using the ablation pen. The left atrial appendage was excluded. By using this modified procedure, we shortened the operation time, simplified the workload of anesthetists, and reduced injury for patients by avoiding thoracotomy, minimizing changes of patient's position during the surgery, and simplifying the trachea cannula management by the anesthetists. In conclusion, we have shown that 
the modified Cox Maze III procedure under VATS has technical advantage and feasible especially to AF patients with IVC anomalies.

The sinus rhythm was restored in the patient after surgery. She has been followed up well for 2 years since the operation with no recurrence of AF. The results indicate that the modified Cox Maze III procedure may be a good option for treatment of paroxysmal or persistent AF, especially to AF patients with IVC anomalies. Of course, this was a single-center study, more cases and further follow-up data should be collected to substantiate our findings.

\section{ACKNOWLEDGEMENT}

This work was supported by a grant from Medical Health Science and Technology Project of Zhejiang Provincial Health Commission (no. 2018ZD024). Written informed consent was obtained from the patient for publication of this report and any accompanying images.

\section{REFERENCES}

Giordano JM, Trout HH III. 1986. Anomalies of the inferior vena cava. J Vasc Surg. 3(6):924-928.

Maxwell EW, Erwin GS. 1928. Four cases of anomalous inferior vena cava with an explanation of their developmental origin. J Anat. 62(pt 2):184-197.

McClure CFW, Butler EG. 1925. The development of the vena cava inferior in man. Am J Anat. 35(3):331-383.

Mei J, Ma N, Ding F, Chen Y, Jiang Z, Hu F, Xiao H. 2014. Complete thoracoscopic ablation of the left atrium via the left chest for treatment of lone atrial fibrillation. J Thorac Cardiovasc Surg. Jan;147(1):242-6.

Sahin H, Pekcevik Y, Aslaner R. 2017. Double Inferior Vena Cava (IVC) With Intrahepatic Interruption, Hemiazygos Vein Continuation, and Intrahepatic Venous Shunt. Vasc Endovascular Surg. Jan;51(1):38-42.

Wolf RK, Schneeberger EW, Osterday R, Miller D, Merrill W, Flege JB $\mathrm{Jr}$, et al. 2005. Video-assisted bilateral pulmonary vein isolation and left atrial appendage exclu- sion for atrial fibrillation. J Thorac Cardiovasc Surg. 130:797-802. 